\title{
Investigation of sleep disorders including home monitoring
}

Many children have a sleep disorder. ${ }^{1}$ Often it is sufficiently frequent or intense for it to seriously concern parents. Particularly high rates are reported for certain groups, such as children with a mental handicap, ${ }^{2}$ and many physical illnesses are likely to be complicated by sleep disruption. ${ }^{3}$

There is, therefore, a need for comprehensive coverage of children's sleep disorders in medical education at undergraduate and postgraduate levels to ensure familiarity with the clinical features of sleep disorders, the conditions with which sleep disorders may be confused, and with the many forms of treatment that are available.

The place of special sleep studies also needs to be more widely known, including the comparatively recent availability of systems by which objective information about sleep patterns and physiology can be obtained from recordings taken at home or in other non-specialised settings.

The range of sleep problems for which such evaluation may be appropriate is wide. ${ }^{4}$ The three main types (of which there are many varieties) are: sleeplessness of one form or another, ${ }^{5}$ episodic disturbances of behaviour (parasomnias), ${ }^{6}$ and excessive sleepiness during the day. ${ }^{7}$

\section{Diagnostic approach and procedures \\ CLINICAL ASSESSMENT}

Reflecting the general neglect of sleep disorders, history taking protocols usually contain few items concerning sleep and need to be supplemented with a systematic review of the 24 hour sleep-wake pattern, and sleep arrangements and environment. A sleep diary is often instructive. Important information may be available not only from parents but older children themselves and sometimes from siblings. Sleep questionnaires can provide a useful framework for detailed clinical inquiry as in recent studies of children with Down's syndrome ${ }^{8}$ or tuberous sclerosis. ${ }^{9}$

\section{OBJECTIVE ASSESSMENT}

Detailed inquiry about sleep patterns and problems should be attempted but parents' recollection of their child's sleep patterns and problems may well be incomplete or distorted. Therefore, in some cases at least, more objective assessment is needed for accuracy or, alternatively, when physiological information is required.

\section{(1) Video monitoring}

For episodic disturbances at night, overnight video recordings alone can be highly instructive. Parents can be encouraged to use their family video systems for recordings at home. The disparities between the findings and the usual clinic descriptions of night time attacks are sometimes striking. Overnight video and sound recordings combined with oximetry may also be used (at least as a screening device) for the detection of sleep related breathing problems. ${ }^{10}$

\section{(2) Conventional nocturnal polysomnography}

A major milestone in the understanding of sleep physiology was the description in the 1950 s of rapid eye movement (REM) sleep and then the four stages of non-rapid eye movement (NREM) sleep, with the occurrence of about four to six NREM/REM cycles throughout the night. These phenomena are based on the recording of the electroencephalogram, electro-oculogram and electromyogram, and the definition of the different stages of sleep in terms of universally agreed criteria. ${ }^{11}$

Results are usually expressed in terms of (a) time spent in each stage of sleep (stages 1 to 4 of NREM sleep, REM sleep, plus time awake), (b) latencies to sleep onset, to the first period of deep NREM sleep, and to the first period of REM sleep, (c) NREM-REM cycles, and (d) the occurrence of nocturnal events. This information is presented in the form of sleep statistics and also a 'hypnogram'. Normative data are available for adults and also for children. ${ }^{12}$ In infants, sleep is described in terms of active, quiet, and indeterminate sleep states because well defined stages have not developed. ${ }^{13}$

For the evaluation of sleep related breathing disorders ${ }^{14}$ additional measures are taken of respiratory effort and airflow, blood oxygenation, and possibly expired carbon dioxide and electrocardiography. Video and sound recording are also usually included. Additional electroencephalography channels can be used if nocturnal epilepsy is suspected.

The accepted objective assessment of daytime sleepiness is the multiple sleep latency test, ${ }^{15}$ which measures the readiness with which the subject falls asleep given repeated opportunities to do so in a quiet darkened room.

\section{Ambulatory polysomnography}

There are few specialised sleep disorders centres outside North America. Such laboratories are very expensive to staff and run and are unacceptable to many patients, especially children. As a result, there is increasing interest in ambulatory sleep recording systems ${ }^{16}$ for use at home, in hospital wards, intensive care units, and other nonspecialised environments.

Ambulatory polysomnography implies recording physiological parameters from subjects free from stationary recording devices. Probably the most widely used system of this type is the Oxford Medilog. ${ }^{17}$ The latest version provides information (recorded onto cassette tape) on basic sleep parameters plus, where necessary, the additional measures described earlier. An automated sleep staging system is available the results of which have been shown to produce acceptable correspondence with visual scoring for normal sleep patterns. ${ }^{18}$

In addition to the lower cost compared with admission to a sleep laboratory, even a single night of home recording may provide a more representative sample of the subject's sleep because of a reduced 'first night effect', that is, sleep pattern disturbance caused by the recording procedure and laboratory environment. ${ }^{19}$ This reduction is thought to result from the subject's familiarity with his own bed, the absence of an observing technician, and adaptation to the equipment provided it is fitted well before bedtime. Long duration recordings are possible over at least one 24 hour sleep wake cycle which is of particular importance for the detection of daytime napping or other events that occur outside the usual hours of sleep. In addition, investigations of sleep wake cycle patterns are possible in naturalistic settings where the effects of sleep disturbing influences of a physical or psychological nature can be studied. 
As the circumstances in which ambulatory recordings are made are non-standardised, with limited control over the subject's activity levels or other factors influencing his sleep-wake patterns, a sleep-wake log completed for the whole recording period is desirable, combined with the use of an event marker built into the cassette recorder. Alternatively, combined video recordings and cassette recordings can be used. The optimal selection of physiological parameters, and number of channels devoted to each, needs to be considered in the light of the particular sleep problem under investigation.

An exact correspondence cannot be expected between the results obtained by ambulatory polysomnography and conventional polysomnography because of the different recording environments. Indeed, for clinical purposes, results obtained at home might well be more relevant to diagnosis and management. Where parallel recordings have been made, results obtained by ambulatory sleep systems have been shown to be generally in agreement with conventional polysomnography. ${ }^{20}$

There is good evidence of the value of ambulatory polysomnography when used with experience and conviction. However, its use with children seems to have been unnecessarily restricted and there is a need for both wider use and further evaluation in young subjects.

Kayed has described the value of this type of procedure in the absence of specialised sleep laboratory facilities. ${ }^{21}$ McCall et al illustrate its selective use in the insomnias, hypersomnias, parasomnias and sleep-wake cycle disorders, ${ }^{22}$ while Broughton focuses on excessive daytime sleepiness. ${ }^{23}$ Opinions differ about the adequacy of home sleep recordings regarding sleep related respiratory problems. The general view seems to be that ambulatory monitoring should be restricted to screening purposes. The place of ambulatory monitoring in the parasomnias has not been systematically evaluated although there are individual case illustrations. ${ }^{24}$ Its role in the investigation of epilepsy, including sleep related seizures, is well established across a wide age range from the neonatal period onwards. ${ }^{25} 26$

Home sleep studies are inappropriate for patients who are uncooperative or medically ill, and in the presence of simultaneous sleep pathologies or where the precise relationships between pathological events and specific stages need to be established. Wherever the sleep recordings are performed, experienced technologists, and staff skilled in the interpretation of the findings, are essential.

\section{Other recent developments}

There is increasing interest in the significance for daytime function of changes in the microstructure of sleep. Frequent brief arousals (characterised mainly by abrupt changes in electroencephalographic frequency, suggestive of the awake state but without actual awakening) 'fragment' sleep without shortening it or changing conventional sleep stages. These 'microarousals' have been associated with impaired cognitive function, mood changes, and complaints of fatigue in a variety of clinical disorders and experimental subjects. ${ }^{27} 28$
At the other extreme, where a detailed account of sleep physiology is not required or possible, monitoring of body movements during sleep can be a simple and inexpensive way of distinguishing between the awake and asleep states. Of the various methods that have been devised, increasing use is being made of small wrist worn movement sensors. Good correspondence with polysomnographic data has been reported at all ages including infants and young children. ${ }^{29}$

GREGORY STORES

University of Oxford Department of Psychiatry,

Park Hospital for Children,

Old Road, Headington,

Oxford $O X 37 L Q$

1 Richman N. Surveys of sleep disorders in children in a general population. In: Guilleminault C, ed. Sleep and its disorders in children. New York: Raven Press, 1987: 115-27.

2 Stores G. Sleep studies in children with a mental handicap. $\mathcal{f}$ Child Psychol Psychiatry 1992; 33: 1303-17.

3 Stores G. Sleep disorders in clinical practice [Editorial]. $\mathrm{Br} f \mathrm{Clin}$ Pract 1992; 46: 82-4

4 Stores G. Sleep disorders. Current Paediatrics 1992; 2: 145-50.

5 Ferber R. The sleepless child. In: Guilleminault C, ed. Sleep and its disorders in children. New York: Raven Press, 1987: 141-63.

6 Mahowald MW, Rosen GM. Parasomnias in children. Pediatrician 1990; 17: $21-31$.

7 Sheldon SH, Spire J-P, Levy HB. Disorders of excessive somnolence. Pediatric sleep medicine. Philadelphia: Saunders, 1992: 91-105.

8 Stores R. Sleep problems in children with Down's syndrome: a summary report. Down's syndrome: Research and Practice 1993; 1: 72-4.

9 Hunt A, Stores G. Sleep disorder and epilepsy in children with tuberous sclerosis: a questionnaire-based study. Dev Med Child Neurol 1994; 36: 108-15.

10 Ali NJ, Pitson DJ, Stradling JR. Snoring, sleep disturbance and behaviour in 4-5 year olds. Arch Dis Child 1993; 68: 360-6.

11 Carskadon $M$, Rechtschaffen A. Monitoring and staging human sleep. In: Kryger MH, Roth T, Dement WC, eds. Principles and practice of sleep medicine. Philadelphia: Saunders, 1989: 665-83.

12 Sheldon SH, Spire J-P, Levy HB. Normal values. Pediatric sleep medicine. Philadelphia: Saunders, 1992: 178-84.

13 Hoppenbrouers T. Sleep in infants. In: Guilleminault C, ed. Sleep and its disorders in children. New York: Raven Press, 1987: 1-15.

14 Stradling JR. Consensus report: sleep studies for sleep-related breathing disorders. Fournal of Sleep Research 1992; 1: 265-73.

15 Carskadon MA, Dement WC, Mittler M, Roth T, Westbrook PR. Keenan F. Guidelines for the multiple sleep latency test (MSLT): a standard measure of sleepiness. Sleep 1986; 9: 519-24.

16 McCall WV, Erwin CW, Edinger JD, Krystal AD, Marsh GR. Ambulatory polysomnography: technical aspects and normative values. $\mathcal{f}$ Clin Neurophysiol 1992; 9: 68-77.

17 Broughton RJ. Ambulant home monitoring of sleep and its disorders. In: Kryger MH, Roth T, Dement WC, eds. Principles and practice of sleep medicine. Philadelphia: Saunders, 1989: 696-701.

18 Höller L, Riemer H. Comparison of visual analysis and automatic sleep stage scoring (Oxford Medilog 9000 system). Eur Neurol 1986; 25 (suppl 2): $36-45$.

19 Sharpley AL, Solomon RA, Cowen PJ. Evaluation of first night effect using ambulatory monitoring and automatic sleep stage analysis. Sleep 1988; 11: 273-6.

20 Sewitch DE, Kupfer DG. Polysomnographic telemetry using Telediagnostic and Oxford Medilog 9000 systems. Sleep 1985; 8: 288-93.

21 Kayed K. Use of home monitoring in a sleep disorders clinic. In: Miles LE, Broughton RJ, eds. Medilog monitoring in the home and work environment. New York: Raven Press, 1990: 245-54.

22 McCall WV, Edinger JD, Erwin CW. Clinical utility of cassette polysomnography in sleep and sleep related disorders. In: Ebersole JS, ed. polysomnography in sleep and sleep related disorders. In: Ebersole JS

23 Broughton RJ. Ambulatory sleep-wake monitoring in the hypersomnias. In: Ebersole JS, ed. Ambulatory EEG monitoring. New York: Raven Press, 1989: 277-98.

24 Stores G. Confusions concerning sleep disorders and the epilepsies in children and adolescents. Br $\mathcal{F}$ Psychiatry 1991; 158: 1-7.

25 Eyre JA. Clinical utility of cassette EEG in neonatal seizure disorders. In: Ebersole JS, ed. Ambulatory EEG monitoring. New York: Raven Press, 1989: 141-55.

26 Stores G, Bergel N. Clinical utility of cassette EEG in childhood seizure disorders. In: Ebersole JS, ed. Ambulatory EEG monitoring. New York: Raven Press, 1989: 129-39.

27 Stepanski E, Lamphere J, Badia P, Zorick F, Roth T. Sleep fragmentation and daytime sleepiness. Sleep 1984; 7: 18-26.

28 Bonnet MH. Effect of sleep disruption on sleep, performance and mood. Sleep 1985; 8: 11-9.

29 Sadeh A, Lavie P, Scher A, Tirosh E, Epstein R. Actigraphic homemonitoring sleep disturbed and control infants and young children: a new method for pediatric assessment of sleep-wake patterns. Pediatrics 1991 87: 494-9. 